\title{
Symmetries and Conservation Laws for Hamiltonian Systems
}

\author{
Estomih Shedrack Massawe \\ Department of Mathematics, University of Dar es Salaam, Dar es Salaam, Tanzania
}

Email address:

emassawe2@gmail.com, estomihmassawe@yahoo.com

To cite this article:

Estomih Shedrack Massawe. Symmetries and Conservation Laws for Hamiltonian Systems. American Journal of Applied Mathematics. Vol. 4, No. 3, 2016, pp. 132-136. doi: 10.11648/j.ajam.20160403.13

Received: April 19, 2016; Accepted: May 3, 2016; Published: May 14, 2016

\begin{abstract}
In this paper, it is shown that symmetry of a physical system is a transformation which may be applied to the state space without altering the system or its dynamical interaction in any way. The theory is applied to generalize the concept of symmetries and conservation laws with external to Hamiltonian systems with external forces. By this we obtain a generalized Noether's Theorem which states that for Hamiltonian systems with external forces, a symmetry law generates a conservation law and vice versa.
\end{abstract}

Keywords: Symmetries, Conservation Laws, Hamiltonian Systems

\section{Introduction}

Symmetries are among the most important properties of dynamical systems when they exist [1]. The study of symmetries is very important in the sense that they are equivalent to the existence of conservation laws. [2] has shown that in Hamiltonian system, symmetries are very close to the constants of the motion. Noether's theorem has also advocated this concept. Also [3] applied symmetries and constants of motion and derived the reduced Hamiltonian system. Generally, symmetry of a physical system is a transformation which may be applied to the state space without altering the system or its dynamical interaction in any way. Consider for example motion of a particle in a central force field with potential $U(|x|)$ where $x$ is the position vector of the particle. This system is not affected by rotations and they are referred to as a symmetry. The existence of such symmetries gives insight into the structure of the system i.e. any solution of the system must reflect these symmetries. Thus it is useful to make use of any symmetry information available in obtaining solutions of the system i.e. constants of the motion (conservation laws) which are defined as mappings $I: T M \rightarrow \mathbb{R}$ such that $d I / d t=0$. Think for example, the energy of the system. It is usually a mapping on the tangent bundle and it is usually constant of the motion. The connection between the symmetry of a system and its corresponding conservation law is summarized in Noether's theorem which follows later. It is therefore intended to formulate and analyse Symmetries and Conservation Laws for Hamiltonian Systems which finally summarized by the generalized Noether's theorem.

\section{Formulation of the Concept of Symmetry}

Let $M$ be the configuration manifold for a physical system. Let $L(q, \dot{q}, t)$ be the Lagrangian of the system i.e. $L: T M \rightarrow \mathbb{R}$ is a smooth function on the tangent bundle $T M$ of the system. Let $h: M \rightarrow M$ be a smooth map on $M$ and $h_{*}: T M \rightarrow T M$ the corresponding bundle map. A Lagrangian $L$ is said to be invariant under the mapping $h$ if $L \circ h_{*}=L$ for any tangent vector $v \in T M$ i.e. $L\left(h_{*} v\right)=L(v)$

[1]. The extension of the symmetry of a physical system to dynamical systems yields the following: [4].

Definition 1

(a) A symmetry for time-invariant external dynamical system $\sum_{e} \subset W^{\mathbb{R}}$ is a map $\psi: W \rightarrow W$ which leaves $\sum_{e}$ invariant i.e. if $w(.) \in \sum_{e}$ then also $\psi(w().) \in \sum_{e}$, and if $w(.) \in \sum_{e}$ then there exists $\tilde{w}(.) \in \sum_{e} \quad$ such that $\psi(\tilde{w}())=.w($.$) . In short$ 
$\psi\left(\sum_{e}\right)=\sum_{e}$

(b) A symmetry for a dynamical system in state space form $\sum_{i} \subset(X \times W)^{\mathbb{R}} \quad$ is $\quad$ a $\quad$ mapping $(\varphi, \psi): X \times W \rightarrow X \times W \quad$ with $\quad \varphi: X \rightarrow X \quad$ and $\psi: W \rightarrow W$ which leaves $\sum_{i}$ invariant i.e. if $(x(),. w().) \in \sum_{i} \quad$ then also $(\varphi(x()),. \psi(w()).) \in \sum_{i}$ and if $(x(),. w().) \in \sum_{i}$ then there exists $(\tilde{\mathbf{x}}(),. \tilde{\mathbf{w}}().) \in \sum_{i}$ such that $(\varphi(\tilde{\mathbf{x}}()),. \psi(\mathbf{w}()))=.(\mathbf{x}(),. \mathbf{w}()$.$) .$

\section{Example}

Consider a particle of mass $m$ moving in $\mathbb{R}^{3}$ subject to a potential $V$ and to which n external force $F$ is applied. The external variables are $F$ and the observation of the position, i.e. $y=r$. If $V$ is invariant under rotations around the $e_{1}$-axis in $\mathbb{R}^{3}$ then the external symmetry $\psi$ is given by simultaneously rotating $y$ and the direction of $F$ around $e_{1}$-axis . The internal symmetry $\varphi$ is given by simultaneously rotating the position and the velocity or momentum around the $e_{1}$-axis [7].

We note that if $(\varphi, \psi)$ is a symmetry for $\sum_{i}$, then $\psi$ is an external symmetry for external behaviour $\sum_{e}$ of $\sum_{i}$.

Accordingly, using differential geometry approach we give the following definition.

Definition 2

Let $\sum(X, W, B, f)$ be a smooth nonlinear system. A symmetry for this system is given by a triplet $(\varphi, \psi, \phi)$ such that $\varphi: X \rightarrow X \quad, \quad \psi: W \rightarrow W \quad, \quad$ and $\phi: B \rightarrow B$ are diffeomorphism for which the following diagram commutes. [4].

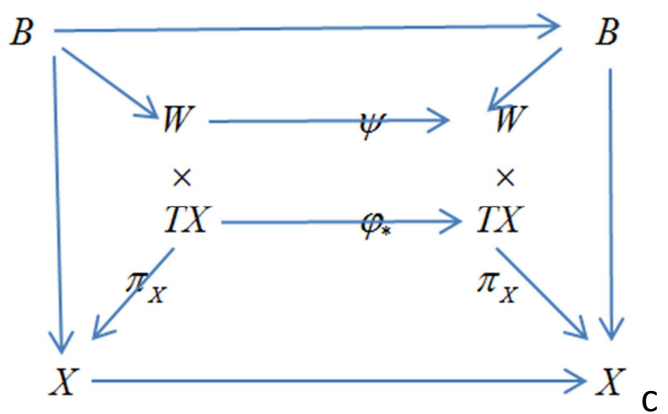

We note that if $(\varphi, \psi, \phi)$ is a symmetry for $\sum(X, W, B, f)$, then $(\varphi, \psi)$ is a symmetry for $\sum_{i}(X, W, B, f)$ and $\psi$ is a symmetry for $\sum_{e}(X, W, B, f)$ in the sense of definition 1 .

Suppose that the symmetry of a physical system is given by a one parameter group of diffeomorphisms $h^{s}$ which leaves the Lagrangian invariant i.e. $L \circ h_{*}^{s}=L$ for all $s$. In the above example of the motion of a particle in a central force field, the parameter $s$ is the rotation angle and the oneparameter group is a group of rotations. Then we have the following definition of infinitesimal symmetries which correspond to 1 and 2 .

Definition 3

Let $\sum(X, W, B, f)$ be a smooth nonlinear system. An infinitesimal symmetry is given by a triple $(S, T, R)$ with $S, T$ and $R$ vectorfields on $X, W$ and $B$ respectively such that $\left(S_{t}, T_{t}, R_{t}\right)$ is a symmetry for every $t \in \mathbb{R}$ and small i.e. if $S_{t}, T_{t}, R_{t}$ are the one-parameter groups generated by $S, T$ and $R$ respectively, then the following diagram commutes. [4].



The consequence of the commutativity of the above diagram is that the one-parameter group $\left(S_{t}, T_{t}\right)$ acting on $X \times W$ takes a feasible state/external signal trajectory into a similar pair.

Since the objective of this paper is to relate symmetries when they exist to conservation laws, we shall next define a conservation law.

\section{Definition 4}

Let $\sum_{i} \subset(X \times W)^{\mathbb{R}}$ be a dynamical system in state space form and let $\sum_{e} \subset W^{\mathbb{R}}$ be its external behaviour. Let $F_{e}: W \rightarrow \mathbb{R}$ be such that for every $w \in \sum_{e}, F_{e}(w().) \in$ locally integrible vector-valued functions on $\mathbb{R}$, and let $F: X \rightarrow \mathbb{R}$. The pair $\left(F, F_{e}\right)$ is called a conservation law if

$$
F\left(x\left(t_{2}\right)\right)-F\left(x\left(t_{1}\right)\right)=\int_{t_{1}}^{t_{2}} F_{e}(w(\tau)) d \tau
$$

Holds for all $(x, w) \in \sum_{i}$ and for all $t_{2} \geq t_{1} . F$ is called the conserved quantity. [4].

The interpretation of equation (1) is that the change of $F$ along a trajectory $x$ is a function of the external trajectory $w$ only.

We use the differential geometry to equation (1). Let $F: X \rightarrow \mathbb{R}$ be a smooth function. Define $F: T X \rightarrow \mathbb{R}$ by $\dot{F}(v)=d F(v)$ for $\mathbf{v} \in T X[4]$.

Definition 5 
Let $\sum(X, W, B, f)$ be a nonlinear dynamical system with $f=(g, h)$ such that $g: B \rightarrow T X$ and $h: B \rightarrow W$. Let $F: X \rightarrow \mathbb{R}$ and $F_{e}: W \rightarrow \mathbb{R}$ be smooth functions. Then the pair $\left(F, F_{e}\right)$ is called a conservation law if $\dot{F} \circ g=F_{e} \circ h$ [4].

If $(x, u)$ are fibre respecting coordinates for $B$, then $\dot{F}(x, \dot{x})=\sum_{i=1}^{n} \frac{\partial F}{\partial x_{i}}$ [5]. Therefore $\dot{F} \circ g(x, u)=\sum_{i=1}^{n} \frac{\partial F}{\partial x_{i}} g_{i}(x, u)$. But $\dot{F} \circ g(x, u)$ is the time derivative of $F$ in $x$ along a trajectory of the vectorfield $g(., u)$. Equation (3) therefore yields $\frac{d}{d t} F(x, u)=F_{e}(h(x, u))$. We note that $\frac{d F}{d t}$ is the Lie derivative $£_{g(.)} F$.

If the external influence to a system is absent then $F_{e}(w(t))=0 \quad \forall w \in \sum_{e}$. The conservation law amounts to $F\left(x\left(t_{1}\right)\right)=F\left(x\left(t_{2}\right)\right) \quad \forall t_{2} \geq t_{1}$ and $\frac{d}{d t} F=0$.

Various laws of conservation are particular cases of Noether's theorem. Noether's theorem relates the symmetries of the configuration manifold of a Lagrangian system to conservation Laws. The consequence of the existence of symmetries is the existence of symmetries of a first integral of the equations of motion. This is the content Noether's theorem and we shall state it. For simplicity only the autonomous case shall be considered.

Theorem 1: (Noether's theorem)

Let $(M, L)$ be a Lagrangian system and let $h^{s}: M \rightarrow M$, $s \in \mathbb{R}$ be a one-parameter group of diffeomophism. If the system $(M, L)$ admits symmetry under the mapping $h^{s}$, thenthe Lagrangian system of equations corresponding to the Lagrangian $L$ has a first integral $I: T M \rightarrow \mathbb{R}$. In local coordinates of $M, I$ is given by $I(q, \dot{q})=\left.\frac{\partial L}{\partial \dot{q}} \frac{\partial h^{s}}{\partial s}(q)\right|_{s=0}$ [4].

Proof

Let $M=\mathbb{R}^{n}$ be the coordinate space. Denote the solution of the Lagrange's equations by $q=\Psi(t)$ where $\Psi: \mathbb{R} \rightarrow M$. It is easy to see that since $h^{s}: M \rightarrow M$, it follows that the Lagrangian $L$ is invariant under the mapping $h_{*}^{s}: T M \rightarrow T M$. Consequently, the mapping $h^{s} \circ \Psi: \mathbb{R} \rightarrow M$ which is just a translation of the solution of the Lagrange's equations for any $s$.

Now define the mapping $\phi: \mathbb{R} \times \mathbb{R} \rightarrow \mathbb{R}^{n}$ by $q=\phi(s, t)=h^{s}(\Psi(t))$. By the hypothesis of invariance of $L$ under the mapping $h^{s}$, we have

$$
0=\frac{\partial L}{\partial s}(\phi, \dot{\phi})=\left.\frac{\partial L}{\partial q} \frac{d \phi}{d s}\right|_{q=\phi(s, t)}+\left.\frac{\partial L}{\partial \dot{q}} \frac{d \dot{\phi}}{d s}\right|_{\dot{q}=\dot{\phi}(s, t)}
$$

The mapping $\left.\phi\right|_{s=\text { constant }}: \mathbb{R} \rightarrow \mathbb{R}^{n}$ for fixed $s$ satisfies
Lagrange's equations

$$
\frac{\partial}{\partial t}\left[\frac{\partial L}{\partial \dot{q}}(\phi(s, t), \dot{\phi}(s, t))\right]=\frac{\partial L}{\partial q}(\phi(s, t), \dot{\phi}(s, t)) .
$$

Define $F(s, t)=\left(\frac{\partial L}{\partial \dot{q}}\right)(\phi(s, t), \dot{\phi}(s, t))$ and substitute $\frac{\partial F}{\partial t}$ for $\frac{\partial L}{\partial q}$ in (2) equation to get

$$
\begin{aligned}
0 & =\left(\frac{d}{d t} \frac{\partial L}{\partial \dot{q}}\right) \frac{d q}{d s}+\frac{\partial L}{\partial \dot{q}}\left(\frac{d}{d t} \frac{d q}{d s}\right) \\
& =\frac{d}{d t}\left(\frac{\partial L}{\partial \dot{q}} \frac{d q}{d s}\right) \\
& =\frac{d I}{d t}
\end{aligned}
$$

\section{Symmetries and Conservation Laws for Hamiltonian Systems}

In this section we specialize the concept of symmetries to Hamiltonian systems. In this case it becomes stronger for the reason that we shall want it to preserve the symplectic structure. Define a symmetry for a Hamiltonian system as follows:

Definition 6

Let $\sum(M, W, B, f)$ be a full Hamiltonian system. An internal symmetry $(\varphi, \psi, \phi)$ is called Hamiltonian if $\varphi$ and $\psi$ are simplectomorphism i.e.

(i) $\varphi^{*} \omega=\omega$

(ii) $\psi^{*} \omega^{e}=\omega^{e}$

with $\varphi^{*}$ and $\psi^{*}$ the pullbacks of $\omega$ and $\omega^{e}$ by $\varphi$ and $\psi$ respectively. [7] has pointed out that for minimal systems we don't have to assume a priori that $\varphi: M \rightarrow M$ is a symplectomorphism. $\varphi$ is implied by the external symmetry $\psi$ as shown by the following proposition.

Proposition 1

Let $\sum(M, W, B, f)$ be a full Hamiltonian and minimal system. Let $(\varphi, \psi, \phi)$ be an internal symmetry and $\psi$ a symplectomorphism. Then $\varphi$ is necessarily also a symplectomorphism [6].

Proof

Let $f=(g, h)$. Because $(\varphi, \psi, \phi)$ is a symmetry, $f(B)$ is mapped by $\psi$ and $\varphi_{*}$ onto $f(B)$ where $\varphi_{*}$ is the derivative map of $\varphi$. Therefore $\sum(M, W, B, \tilde{f})$ with $\tilde{f}=\left(\varphi_{*} \circ g, \psi \circ h\right)$ is again a Hamiltonian system. Hence 
$g^{*}\left(\left(\varphi_{*}^{*}\right)^{*} \dot{\omega}\right)=h^{*}\left(\varphi^{*} \omega^{e}\right)$ and $g^{*} \dot{\omega}=h^{*} \omega^{e}=h^{*} \psi^{*} \omega^{e}=g^{*}\left(\left(\varphi_{*}\right)^{*} \dot{\omega}\right)$ where we have used $\psi^{*} \omega^{e}=\omega^{e}$. This yields $g^{*} \dot{\bar{\omega}}=0$ with $\bar{\omega}=\omega-\varphi^{*} \omega$. [4] has derived that $\sum$ satisfies the minimality rank condition, then $\bar{\omega}=0$ and $\varphi^{*} \omega=\omega$.

We shall now consider the case of the infinitesimal symmetries for Hamiltonian systems.

A vectorfield $S$ on a symplectic manifold $(M, \omega)$ is called a symmetry for Hamiltonian vectorfield $X_{H}$ on $M$ if [6].

(i) The Lie derivative $£_{S} \omega=0$,

(ii) $S(H)=0$ where $H$ is the Hamiltonian function.

From (i) it follows that $S$ has locally a corresponding Hamiltonian function $F: M \rightarrow \mathbb{R}$ and so (ii) implies that $X_{H}(F)=0$ and therefore $F$ is a conserved quantity for $X_{H}$. Conversely for $F: M \rightarrow \mathbb{R}$ such that $X_{H}(F)=0$ it follows that $S=X_{F}$ satisfies (i) and (ii) and so $S$ is a Hamiltonian symmetry.

The generalization of the above to the Hamiltonian system yields the following definition:

\section{Definition 7}

Let $\sum(M, W, B, f)$ be a full Hamiltonian system. An infinitesimal symmetry $(R, S, T)$ for $\sum$ is called Hamiltonian if $S$ and $T$ are locally Hamiltonian vectorfields i.e. $\mathfrak{f}_{S} \omega=0$ and $\mathfrak{£}_{T} \omega^{e}=0$ [9].

A conservation law for a Hamiltonian system can be constructed in the following way:

Consider a Hamiltonian system with an input $u$. For every $u$ we get a Hamiltonian vectorfield on $M$ denoted by $X^{u}$. If $(R, S, T)$ is a Hamiltonian symmetry for $\sum(M, W, B, f)$ then there exists functions $F: M \rightarrow \mathbb{R}$ and $F^{e}: M \rightarrow \mathbb{R}$ with $S=X_{F}$ and $T=X_{F^{e}}$ such that $\forall x \in M$ and $(x, u) \in B, X^{u}(F)(x)=F^{e}(h(x, u))$ where $(g, h)=f: B \rightarrow T M \times W$ [9] We note that $S=X_{F}$ and $T=X_{F^{e}}$ implies that $F$ and $F^{e}$ are Hamiltonian functions. The pair $\left(F, F^{e}\right)$ is the conservation law for the Hamiltonian system $\sum(M, W, B, f)$.

The interpretation of the above construction is that the change of $F$ along the trajectories of the system is a function of the external variables. Knowledge of the external variables together with the initial conditions can determine the behaviour of $F$ as a function of time.

We conclude with the generalized Noether's theorem.

Theorem 2: (Generalized Noether's theorem)

Let $(R, S, T)$ be an infinitesimal symmetry for a full Hamiltonian system $\sum(M, W, B, f)$. Then locally there exists a conservation law $\left(F, F^{e}\right)$. Conversely if $\left(F, F^{e}\right)$. is a conservation law, then there exists a Hamiltonian symmetry $(R, S, T)$ such that $S=X_{F}$ and $T=X_{F^{e}}$ [4]

The following proposition will be needed for the proof of Noether's theorem.

Proposition 2

Let $\sum(M, W, B, f)$ be a nonlinear dynamical system with $f=(g, h)$.Then $(R, S, T)$ is an infinitesimal symmetry iff
i. $\quad g_{*} R=\dot{S}$
ii. $h_{*}=T$.

$g_{*}$ and $h_{*}$ are derivative maps of $g$ and $h$ respectively [10].

Proof

We note that $(R, S, T)$ is an infinitesimal symmetry iff diagram (1) commutes for every $\left(R_{t}, S_{t}, T_{t}\right)$ with $t$ small. This is equivalent to

(a) $S_{t} \circ g=g \circ R_{t}$

(b) $T_{t} \circ h=h \circ R_{t}$

Differenting (a) and (b) with respect to $t$ at $t=0$ we get (i) and (ii).

Now we proceed with the Generalized Noether's theorem.

For a Hamiltonian system $\sum(M, W, B, f)$ we have $g^{*} \dot{\omega}=h^{*} \omega^{e}$ (By proof of Proposition 1)

$$
\begin{gathered}
\Rightarrow g^{*} \dot{\omega}(R,-)=h^{*} \omega^{e}(R,-), \\
\Leftrightarrow \dot{\omega}\left(g_{*} R, g_{*}-\right)=\omega^{e}\left(h_{*} R, h_{*}-\right),(\text { By proposition 2) } \\
\Leftrightarrow d \dot{F}\left(g_{*}-\right)=d F^{e}\left(h_{*}-\right), \\
\Leftrightarrow d(\dot{F} \circ g)=d\left(F^{e} \circ h\right) .
\end{gathered}
$$

We have used the fact that $\dot{\omega}(\dot{S},-)=d F$ when $\omega(S,-)=d F[4]$. We have thus obtained

$$
\dot{F} \circ g=F^{e} \circ h .
$$

Therefore $\left(F, F^{e}\right)$ is a conservation law. (c.f. definition 4) Conversely:

Let $\left(F, F^{e}\right)$ be a conservation law. This is equivalent to $\dot{F}-F^{e}$ restricted to $f(B)$ equal to zero. For $(x, \dot{x}) \in T M$ and $w \in W$ we set $\left(\dot{F}-F^{e}\right)(x, \dot{x}, w)=\dot{F}(x, \dot{x})-F^{e}(w)$ such that $\dot{F}-F^{e}: T M \times W \rightarrow \mathbb{R} . \dot{F}-F^{e}$ is therefore a Hamiltonian function. Hence we can define the Hamiltonian vectorfield $X_{F^{e}-F}$ on the symplectic manifold $\left(T M \times W, \pi_{1}^{*} \dot{\omega}-\pi_{2}^{*} \omega^{e}\right)$. With $X_{\dot{F}}$ the Hamiltonian vectorfield on $T M$ and $X_{F^{e}}$ the Hamiltonian vectorfield on 
$W$ we have

$X_{\dot{F}-F^{e}}=\left(X_{\dot{F}}, X_{F^{e}}\right)$. Because $\dot{F}-F^{e}$ restricted to $f(B)$ is zero, it follows that $\pi_{1}^{*} \dot{\omega}-\pi_{2}^{*} \omega^{e}\left(X_{k}, X_{\dot{F}-F^{e}}\right)=X_{k}\left(\dot{F}-F^{e}\right)=0$ on $f(B)$ for all Hamiltonian vectorfield $X_{k}$ tangent to $f(B)$. $X_{\dot{F}-F^{e}}=\left(X_{\dot{F}}, X_{F^{e}}\right)$ is also tangent to $f(B)$ since $f(B)$ is Lagrangian. If we denote $X_{F}$ by $S$ and $X_{F^{e}}$ by $T$ then we say $(\dot{S}, T)$ is tangent to $f(B)$ and for $t$ small we obtain

$$
\left(S_{t^{*}}, T_{t}\right) f(B)=f(B)
$$

We construct a Hamiltonian symmetry $(R, S, T)$ by defining a 1-parameter family $\phi_{t}: B \rightarrow B$ such that $\left(S_{t^{*}}, T_{t}\right) \circ f=f \circ \phi_{t}$ for $t$ small and a vectorfield $R$ on $B$ by $R(\mathbf{x})=\left.\frac{d \phi_{t}}{d t}\right|_{t=0}(\mathbf{x})$.

\section{Conclusion}

The concept of symmetry for Hamiltonian systems has been formulated and analysed. It was shown that symmetry of a physical system is a transformation which may be applied to the state space without altering the system or its dynamical interaction in any way. Symmetries and conservation laws with external to Hamiltonian systems with external forces has been analysed. The conservation law for a Hamiltonian system was constructed and which was concluded by generalized Noether's theorem.

\section{References}

[1] V. I. Arnold, Mathematical Methods of Classical Mechanics (Translation of the 1974 Russian Edition), Springer, New York, 1978.

[2] C. M. Marle, On Symmetries and Constants of Motion in Hamiltonian Systems with Non-Holonomic Constraints.

[3] J. E. Marsden and A. Weinstern, Reduction of symplectic manifolds with symmetry, Reports on Mathematical Physics, 5, pp. 121-130, 1974.

[4] A. J. Van der Schaft, Symmetries and Conservation Laws for Hamiltonian Systems with Inputs and Outputs: A Generalization of Noethers Theorem, Systems \& Control Letters, Vol 1, pp. 108-115, 1981.

[5] L. P. Landau and E. M. Lifschitz, Mechanics, Pergamon Press, Oxford, 1976

[6] A. J. Van der Schaft, System Theoretic Description of Physical System, Doctoral Thesis, Mathematical Centrum, Amsterdam, 1984.

[7] V. I. Arnold and S. P. Novikov, Dynamical Systems IV, Springer-Verlag, Berlin, Heidelberg, 1990.

[8] A. J. Van der Schaft, Symmetries, Conservation Laws and Time Reveribility for Hamiltonian Systems with External Forces, Journal of Mathematical Physics, Vol 24, pp. 20952101.

[9] J. C. Willems and A. J. Van der Schaft, Modelling of Dynamical Systems Using External and Internal Variables with Application to Hamiltonian Systems, Dynamical Systems and Microphysics, pp. 233-263, Academic Press, New York, 1982 .

[10] S. P. Banks, Mathematical Theories of Nonlinear Systems, Prentice Hall, New York, 1988. 